Research Article

\title{
New Numerical Algorithm to Solve Variable-Order Fractional Integrodifferential Equations in the Sense of Hilfer- Prabhakar Derivative
}

\author{
MohammadHossein Derakhshan \\ Industrial Engineering Department, Apadana Institute of Higher Education, Shiraz, Iran \\ Correspondence should be addressed to MohammadHossein Derakhshan; m.h.derakhshan.20@gmail.com
}

Received 12 September 2020; Revised 28 January 2021; Accepted 10 February 2021; Published 24 February 2021

Academic Editor: Gaston Mandata N gu r kata

Copyright (c) 2021 MohammadHossein Derakhshan. This is an open access article distributed under the Creative Commons Attribution License, which permits unrestricted use, distribution, and reproduction in any medium, provided the original work is properly cited.

\begin{abstract}
In this article, a numerical technique based on the Chebyshev cardinal functions (CCFs) and the Lagrange multiplier technique for the numerical approximation of the variable-order fractional integrodifferential equations are shown. The variable-order fractional derivative is considered in the sense of regularized Hilfer-Prabhakar and Hilfer-Prabhakar fractional derivatives. To solve the problem, first, we obtain the operational matrix of the regularized Hilfer-Prabhakar and Hilfer-Prabhakar fractional derivatives of CCFs. Then, this matrix and collocation method are used to reduce the solution of the nonlinear coupled variable-order fractional integrodifferential equations to a system of algebraic equations which is technically simpler for handling. Convergence and error analysis are examined. Finally, some examples are given to test the proposed numerical method to illustrate its accuracy and efficiency.
\end{abstract}

\section{Introduction}

Fractional calculus as a branch of mathematical analysis due to important applications in computational science, engineering [1-4], chemistry, and many physical equations such as Burger's equation [5] and Korteweg-de Vries equation [6], and viscous fluid [7] has been broadly significant. Recently, obtaining solution of the linear and nonlinear differential equations has important roles in fractional calculus $[8,9]$, because the behavior of the equations is determined by their analytical solutions. In this paper, a differential equation including a variable-order fractional operator is considered in which the equation is an extension of the fractional differential equations of fractional order. Getting exact solutions of the equations in mathematical sciences and physics is not easy, so approximate solutions to these equations using numerical methods are obtained. Different numerical methods for obtaining solutions of the linear and nonlinear equations in the papers are presented. For instance, in [10], a solution of the nonlinear variable-order fractional Riccati differential equations was obtained by using the spectral technique. A finite difference method for getting approximate solution of the variable-order fractional Volterra integrodifferential equations (VO-FVIDEs) has been considered in [11]. The numerical solution of the variable-order fractional integrodifferential equation consisting of cubic spline interpolation can be obtained in [12]. In paper [13], a collocation technique to obtain solution of the variable-order nonlinear cable equation was found. Obtaining the numerical solution of a class of nonlinear variable-order fractional differential equations (FDEs) by using the Legendre wavelets in [14] is studied. Also, Bhrawy and Zaky [15] used the shifted Jacobi polynomials to obtain the solution for variable-order fractional Schrödinger equations, Bhrawy and Zaky [16] studied the Jacobi-Gauss-Lobatto collocation method to obtain the solution for variable-order fractional 
Schrödinger equations, Mahmoud et al. [17] proposed the Jacobi wavelet collocation method to obtain the solution for variable-order fractional equations, Zaky et al. [18] proposed the shifted Chebyshev polynomials to obtain the solution for variable-order fractional equations, and in [19], a proper discrete form of fractional Gronwalltype inequality is introduced and other methods such as the shifted Jacobi collocation method [13] and shifted Jacobi method [20]. Other methods are proposed to obtain numerical solutions of the variable-order fractional integrodifferential equations in the literatures [21-27]. In this paper, we obtain the solution for the following fractional differential equation of order $0<\mu(t), v \leq 1$ by using of the Chebyshev cardinal functions (CCFs) and the Lagrange multiplier method in which $f(x, t)$ is known and $u(x, t)$ is unknown:

$$
C_{\mathfrak{D}_{\rho, \omega, 0^{+}}^{\gamma, \mu(t), v}} u(x, t)+\mathfrak{D}_{\rho, \omega, 0^{+}}^{\gamma, \mu(t), v} u(x, t)=f(x, t, u(x, t)) .
$$

The initial condition for this equation is defined by

$$
u(x, 0)=d
$$

Here, ${ }^{C} \mathfrak{D}_{\rho, \omega, 0^{+}}^{\gamma, \mu(t), v}$ and $\mathfrak{D}_{\rho, \omega, 0^{+}}^{\gamma, \mu(t), v}$ are regularized HilferPrabhakar fractional derivative [28] and Hilfer-Prabhakar fractional derivative [28] of order $0<\mu(t) \leq 1,0<v \leq 1$, respectively. According to the definition of the Hilfer fractional derivative, this Hilfer-Prabhakar fractional derivative is defined but instead of Riemann integral operator in the Hilfer fractional derivative, Prabhakar integral operator [28] of variable-order fractional is replaced, in which this Prabhakar integral operator for $0<\mu(t) \leq 1$ is given by

$$
\left(\mathbf{E}_{\rho, \mu(t), \omega, 0^{+}}^{\gamma} u\right)(x, t)=\int_{0}^{t}(t-\tau)^{\mu(t)-1} E_{\rho, \mu(t)}^{\gamma}\left(\omega(t-\tau)^{\rho}\right) u(x, \tau) d \tau,
$$

where $E_{\rho, \mu(t)}^{\gamma}\left(\omega t^{\rho}\right)$ is the Prabhakar function and it is a generalization of the two-parametric Mittag-Leffler function $E_{\alpha, \beta}(t), \alpha>0, \beta \in \mathbb{C}$, for $\gamma=1$ and the classical Mittag-Leffler function $E_{\alpha}(t), \alpha>0$, for $\gamma=\mu(t)=1$ is considered which is defined by [28]

$$
\begin{gathered}
E_{\rho, \mu(t)}^{\gamma}(t)=\sum_{n=0}^{\infty} \frac{(\gamma)_{n}}{n ! \Gamma(\rho n+\mu(t))} t^{n}, \\
\mathfrak{R}(\rho), \mathfrak{R}(\mu(t))>0, \\
\gamma>0, \\
t \in \mathbb{C}
\end{gathered}
$$

where $(\gamma)_{n}=(\Gamma(\gamma+n)) / \Gamma(\gamma)$ and $\Gamma($.$) are the gamma$ function; moreover, this Prabhakar function for $\mathfrak{R}(\mu(t))$ $>0$ is an entire function [29]. Our interest in this type of fractional derivative is related to its application in physical phenomena, for example, linear viscoelastic con- stitutive equations [30], anomalous dielectric relaxation of Havriliak-Negami function [31, 32], fractional viscoelasticity [33], spherical stellar systems, stochastic processes [34], telegraph equations [34], and anomalous relaxation in dielectrics [35]. Since this mathematical system which is given in Equation (1), due to the variableorder fractional operators and nonlinearity, is very complex, we need to reduce it by using a highly accurate and efficient expansion scheme. Thus, for this aim, we first extract an operational matrix of variable-order fractional derivatives for the CCFs; then, we use them for extending the unknown solution. In other words, the problem shown in Equation (1) is converted into an algebraic system of equations by exploiting the operational matrix of variable-order derivative. In this paper, we consider a class of variable-order fractional integrodifferential equations. For solving the given equations, operational matrices based on the CCFs are applied. First, we approximate the unknown function and its derivatives in terms of the CCFs. Then, by substituting these approximations into the equation and applying the properties of the CCFs together with the collocation points, the main problem is reduced to a set of nonlinear algebraic equations. By solving this system, the approximate solution is calculated. This article is divided into four sections as follows. In Section 2, some important and application definitions, lemma, and theorem which are applied in the next section are expressed. In Section 3, properties of the Chebyshev cardinal functions (CCFs) and the Lagrange multipliers are introduced. In Section 4, we use the properties described in Section 3 to solve the variable-order fractional differential equations. In Section 5, to illustrate the efficiency and accuracy of the proposed method in this article, four examples are displayed.

\section{Definition and Fundamental Properties of Fractional Calculus}

This section refers to the definitions and lemmas which are used for the next sections, and these definitions and lemmas are taken from articles $[36,37]$.

$$
\mathfrak{D}_{\rho, \omega, 0^{+}}^{\gamma, \mu(t), v} u(x, t)=\left[\mathbf{E}_{\rho,(1-\mu(t)) v, \omega, 0^{+}}^{-v \gamma} \frac{d}{d t}\left(\mathbf{E}_{\rho,(1-\mu(t))(1-v), \omega, 0^{+}}^{-(1-v) \gamma} u(x, t)\right)\right],
$$

where $u(x, t) \in C\left(\left[x_{\min }, x_{\max }\right] \times\left[0, t_{\max }\right]\right)$ and $(d / d t) u(x, t)$ is an absolutely continuous function. Also, the HilferPrabhakar derivative of a regularized type with similar properties above is defined as [37]

$$
C_{\mathfrak{D}_{\rho, \omega, 0^{+}}^{\gamma, \mu(t), v}} u(x, t)=\mathbf{E}_{\rho, 1-\mu(t), \omega, 0^{+}}^{-\gamma} \frac{d}{d t}(u(x, t)) .
$$

Definition 1. For $0<\mu(t) \leq 1,0<v \leq 1, \rho>0$ and $\omega, \gamma \in \mathbb{R}$, the Hilfer-Prabhakar derivative can be defined as $[28,37]$ 
Lemma 2. For $0<\mu(t) \leq 1,0<v \leq 1, \rho>0$ and $\omega, \gamma \in \mathbb{R}$, the following formula holds [36]:

$$
\left(\mathbf{E}_{\rho, \mu(t), \omega, 0^{+}}^{\gamma} t^{q-1}\right)(x)=\Gamma(q) x^{\mu(t)+q-1} E_{\rho, \mu(t)+q}^{\gamma}\left(\omega x^{\rho}\right), \quad q>1 .
$$

Also, the following relation is obtained for fractional operator $\mathfrak{D}_{\rho, \omega, 0^{+}}^{\gamma, \mu(t), v}$ on the function $t^{q-1}[37]$ :

$$
\left(\mathfrak{D}_{\rho, \omega, 0^{+}}^{\gamma, \mu(t), v} t^{q-1}\right)(x)=x^{q-\mu(t)-1} E_{\rho, q-\mu(t)}^{-\gamma}\left(\omega x^{\rho}\right), \quad q>1 .
$$

Lemma 3. For $0<\mu(t) \leq 1,0<v \leq 1, \rho>0$ and $\omega, \gamma \in \mathbb{R}$, the Hilfer-Prabhakar derivative of a regularized model is calculated as follows:

$$
\left({ }^{C} \mathfrak{D}_{\rho, \omega, 0^{+}}^{\gamma, \mu(t), v} t^{q-1}\right)(x)=\Gamma(q-2) x^{q-\mu(t)-1} E_{\rho, q-\mu(t)}^{-\gamma}\left(\omega x^{\rho}\right), \quad q>2 .
$$

Proof. Applying the normal derivative definition and formulas (6) and (7) on the function $t$ with the power of $q$, the result is obtained.

\section{Approximation Function and Properties of the Lagrange and the CCF Polynomials}

Any function $u(x, t) \in C\left(\left[x_{\min }, x_{\max }\right] \times\left[0, t_{\max }\right]\right)$ can be approximated in terms of Chebyshev cardinal functions of order $m$ and Lagrange interpolating polynomials of order $n$ as follows:

$$
u(x, t) \simeq \sum_{i=1}^{m+1} \sum_{j=1}^{n+1} u\left(x_{i}, t_{j}\right) \varphi_{i}(x) \psi_{j}(t)
$$

where $\varphi_{i}(x)$ is the Chebyshev cardinal functions of order $m$ on the interval $x \in\left[x_{\min }, x_{\max }\right]$ which is defined by [38]

$$
\begin{gathered}
\varphi_{i}(x)=\prod_{k=1, k \neq i}^{m+1}\left[\frac{x-x_{k}}{x_{i}-x_{k}}\right], \quad i=1,2, \cdots, m+1, \\
x_{k}=x_{\min } \cos \left(\frac{(2 k-1) \pi}{2 m+2}\right)=\frac{\left(x_{\max }+x_{\min }-\left(x_{\max }-x_{\min }\right)\right)}{2} \cos \left(\frac{(2 k-1) \pi}{2 m+2}\right),
\end{gathered}
$$

and $\psi_{j}(t)$ is the Lagrange interpolating polynomials of order $n$ on the interval $\left[0, t_{\max }\right]$ which is given by [38]

$$
\psi_{j}(t)=\prod_{k=1, k \neq j}^{n+1}\left[\frac{t-t_{k}}{t_{j}-t_{k}}\right], j=1,2, \cdots, n+1,
$$

where

$$
\psi_{j}\left(t_{i}\right)=\delta_{j, i}= \begin{cases}1, & i=j \\ 0, & i \neq j .\end{cases}
$$

Also, in relation (10), $t_{j}$ is considered

$$
t_{j}=\frac{t_{\max }}{2}\left(1-\cos \left(\frac{(2 j-1) \pi}{2 n+2}\right)\right) .
$$

Function $u(x, t)$ in Equation (10) can be rewritten as follows:

$$
u(x, t) \simeq \sum_{i=1}^{m+1} \sum_{j=1}^{n+1} u\left(x_{i}, t_{j}\right) \varphi_{i}(x) \psi_{j}(t) \triangleq Y_{m}^{T}(x) \times \Delta \times \Lambda_{n}(t)
$$

where $Y_{m}(x), \Delta, \Lambda_{n}(t)$ is defined by

$$
\begin{aligned}
Y_{m}(x) & =\left[\varphi_{1}(x), \varphi_{2}(x), \cdots, \varphi_{m+1}(x)\right]^{T}, \\
\Delta & =\left[u_{i j}\right]_{(m+1) \times(n+1)}, \\
\Lambda_{n}(t) & =\left[\psi_{1}(t), \psi_{2}(t), \cdots, \psi_{n+1}(t)\right]^{T} .
\end{aligned}
$$

The purpose of this paper is to express the function $\psi_{j}(t), j=0,1,2, \cdots, n+1$, as an operational matrix as follows [39]:

$$
\begin{aligned}
\psi_{j}(t) & =\frac{1}{\gamma_{j}} \times\left[b_{j, 0} t^{n}+b_{j, 1} t^{n-1}+\cdots+b_{j, n}\right] \\
& =\frac{1}{\gamma_{j}} \times\left[b_{j, 0}, b_{j, 1}, \cdots, b_{j, n}\right]_{1 \times(n+1)} \times\left[\begin{array}{c}
t^{n} \\
t^{n-1} \\
\vdots \\
1
\end{array}\right]_{(n+1) \times 1},
\end{aligned}
$$

where $\gamma_{j}, b_{j, k}$ for $k=0,1,2, \cdots, n$ are given by

$$
\begin{gathered}
\gamma_{j}=\prod_{r=1, r \neq j}^{n+1} t_{j}-t_{r}, \\
b_{j, 0}=1, \\
b_{j, k}=\frac{-\sum_{r=1}^{k} \sum_{l=1}^{n+1} a_{j, r} b_{j, k-r} t_{l}^{r}}{k}, \\
1 \leq k \leq n, \\
r \neq j .
\end{gathered}
$$



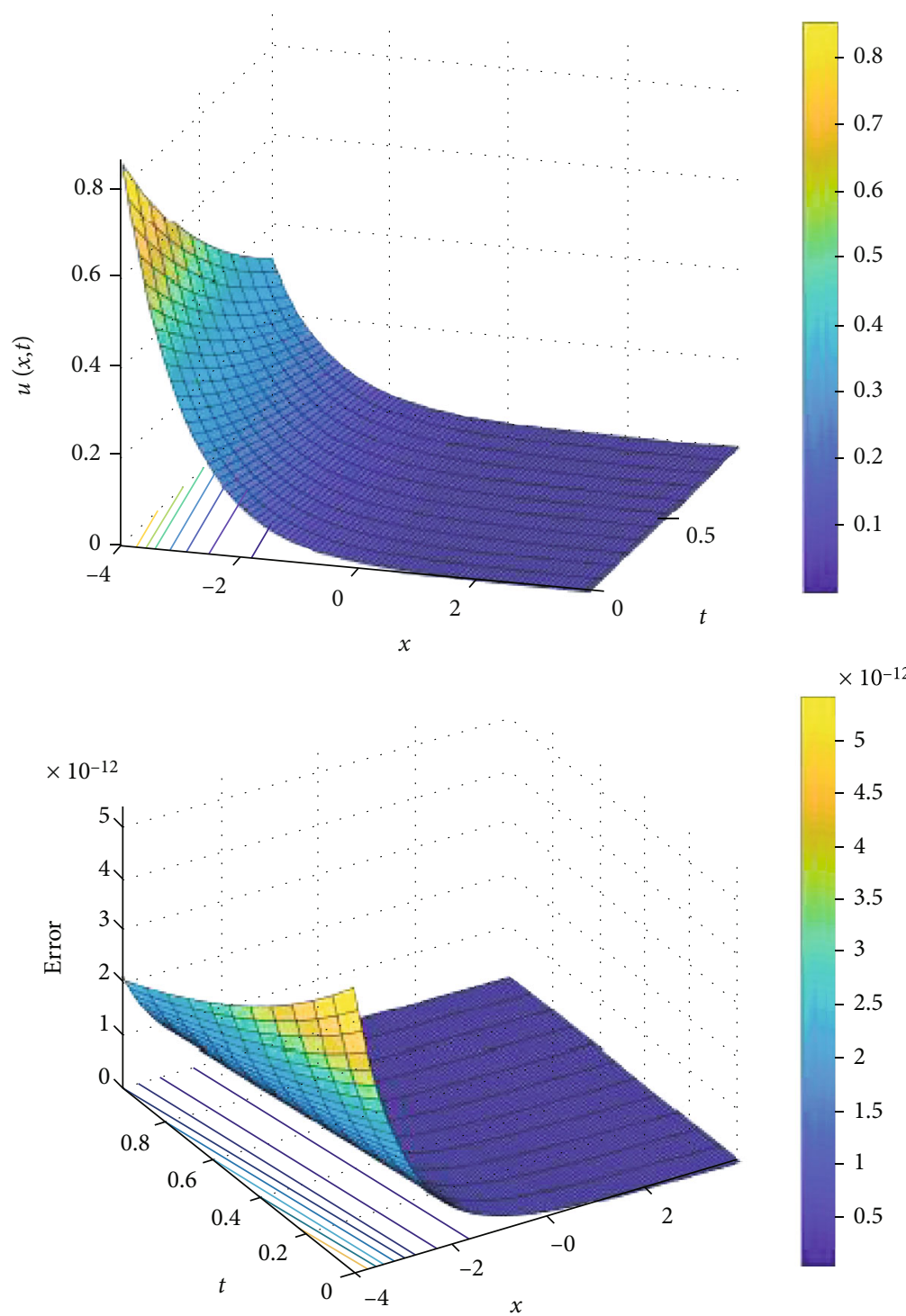

Figure 1: Portrait of the numerical solution and the absolute error for Example 6 with $m=4, n=10$.

TABLe 1: Absolute error for different values of $m, n$ for Example 6.

\begin{tabular}{lcccc}
\hline$(x, t)$ & \multicolumn{2}{c}{$|u(x, t)-u *(x, t)|$} & \multicolumn{2}{c}{$|u(x, t)-u *(x, t)|$} \\
& $m=4, n=10$ & $m=10, n=20$ & $m=15, n=30$ & $4.5778 e-8$ \\
$(0.68,0.09)$ & $4.9947 e-6$ & $5.0090 e-7$ & $1.8612 e-7$ & $3.8237 e-9$ \\
$(0.86,0.18)$ & $4.1719 e-6$ & $4.1838 e-7$ & $1.5546 e-7$ & $3.1938 e-9$ \\
$(1.04,0.27)$ & $3.4846 e-6$ & $3.4946 e-7$ & $1.2985 e-7$ & $2.4381 e-9$ \\
$(1.31,0.09)$ & $2.6601 e-6$ & $2.6677 e-7$ & $9.9127 e-8$ & $9.9127 e-10$ \\
$(2.21,0.36)$ & $1.0815 e-6$ & $1.0846 e-7$ & $4.8250 e-8$ & $4.0302 e-10$ \\
$(3.11,0.45)$ & $4.3971 e-7$ & $4.4097 e-8$ & $1.96172 e-8$ & \\
\hline
\end{tabular}

TABLE 2: $C$-order of the presented method for Example 6.

\begin{tabular}{cccc}
\hline \multicolumn{2}{c}{$C$-order } & \multicolumn{2}{c}{$C$-order } \\
$m=4, n=10$ & $m=10, n=20$ & $m=15, n=30$ & $m=20, n=40$ \\
\hline 8.56 & 9.23 & 11.54 & 13.65 \\
\hline
\end{tabular}

Theorem 4. Suppose $\psi_{j}(t)$ is relation (17). Then, the Hilfer-Prabhakar derivative of a regularized type and the Hilfer-Prabhakar derivative on the function $\psi_{j}(t)$ are obtained, respectively, as follows: 


$$
\begin{aligned}
{ }_{\mathcal{D}_{\rho, \omega, 0^{+}}}^{\gamma, \mu(t), v} \psi_{j}(t) & =\frac{1}{\gamma_{j}} \times\left[b_{j, 0}, b_{j, 1}, \cdots, b_{j, n}\right]_{1 \times(n+1)} \times\left[\begin{array}{c}
\Gamma(n-1) t^{n-\mu(t)} E_{\rho, n-\mu(t)+1}^{-\gamma}\left(\omega t^{\rho}\right) \\
\Gamma(n-2) t^{n-\mu(t)-1} E_{\rho, n-\mu(t)}^{-\gamma}\left(\omega t^{\rho}\right) \\
\vdots \\
0
\end{array}\right]_{(n+1) \times 1} \\
& =\frac{1}{\gamma_{j}} \sum_{k=0}^{n-1} b_{j, k}(n-k-1) ! t^{n-\mu(t)-k} E_{\rho, n-\mu(t)-k+1}^{-\gamma}\left(\omega t^{\rho}\right),
\end{aligned}
$$

$$
\begin{aligned}
\mathfrak{D}_{\rho, \omega, 0^{+}}^{\gamma, \mu(t),} \psi_{j}(t) & =\frac{1}{\gamma_{j}} \times\left[b_{j, 0}, b_{j, 1}, \cdots, b_{j, n}\right]_{1 \times(n+1)} \times\left[\begin{array}{c}
t^{n-\mu(t)} E_{\rho, n-\mu(t)+1}^{-\gamma}\left(\omega t^{\rho}\right) \\
t^{n-\mu(t)-1} E_{\rho, n-\mu(t)}^{-\gamma}\left(\omega t^{\rho}\right) \\
\vdots \\
t^{-\mu(t)} E_{\rho, 1-\mu(t)}^{-\gamma}\left(\omega t^{\rho}\right)
\end{array}\right]_{(n+1) \times 1} \\
& =\frac{1}{\gamma_{j}} \sum_{k=0}^{n} b_{j, k} t^{n-\mu(t)-k} E_{\rho, n-\mu(t)-k+1}^{-\gamma}\left(\omega t^{\rho}\right) .
\end{aligned}
$$

Proof. By taking the Hilfer-Prabhakar derivative of a regularized type on both sides of Equation (17), we get

$$
\begin{aligned}
& C_{\mathfrak{D} p, \omega, 0^{+}}^{\gamma, \mu(t), v} \psi_{j}(t)=\frac{1}{\gamma_{j}} \times\left[b_{j, 0} C_{\mathfrak{D}_{\rho, \omega, 0,0}^{\gamma, \mu(t), v}}\left(t^{n}\right)+b_{j, 1} C_{\mathfrak{D}_{\rho, \omega, 00^{+}}}^{\gamma, \mu(t), v}\left(t^{n-1}\right)+\cdots+b_{j, n} C_{\mathfrak{D} \rho, \omega, 0^{+}}^{\gamma, \mu(t), v}(1)\right] \\
& =\frac{1}{\gamma_{j}} \times\left[b_{j, 0}, b_{j, 1}, \cdots, b_{j, n}\right]_{1 \times(n+1)} \times\left[\begin{array}{c}
C_{\mathfrak{D} \rho, \omega, 0^{+}}^{\gamma, \mu(t), v}\left(t^{n}\right) \\
C_{\mathfrak{D} \rho, \omega, 0^{+}}^{\gamma, \mu(t), v}\left(t^{n-1}\right) \\
\vdots \\
C_{\mathfrak{D} \rho, \omega, 0^{+}}^{\gamma \mu \mu(t), v}(1)
\end{array}\right]_{(n+1) \times 1},
\end{aligned}
$$

applying Equation (9) on Equation (21) and the result is achieved. Prove relation (20) like the same process is considered for the Hilfer-Prabhakar derivative of a regularized type.

Now, using Theorem 4, the function Hilfer-Prabhakar derivative of a regularized-type $\psi_{j}(t)$ can be approximated as a finite series in the form of the Chebyshev cardinal functions as follows:

$$
\begin{aligned}
C_{\mathfrak{D}_{\rho, \omega, 0^{+}}^{\gamma, \mu(t), v} \psi_{j}(t)} & \simeq \sum_{r=1}^{n+1}\left[\frac{1}{\gamma_{j}} \sum_{k=0}^{n-1} b_{j, k}(n-k-1) ! t_{r}^{n-\mu(t)-k} E_{\rho, n-\mu\left(t_{r}\right)-k+1}^{-\gamma}\left(\omega t_{r}^{\rho}\right)\right] \psi_{r}(t) \\
& =\sum_{r=1}^{n+1} \mathbb{q}_{j, r}^{\mu(t)} \psi_{r}(t), \quad j=1,2, \cdots, n+1 .
\end{aligned}
$$

Also, using Theorem 4, the function Hilfer-Prabhakar derivative $\psi_{j}(t)$ can be approximated as a finite series in the form of the Chebyshev cardinal functions as follows:
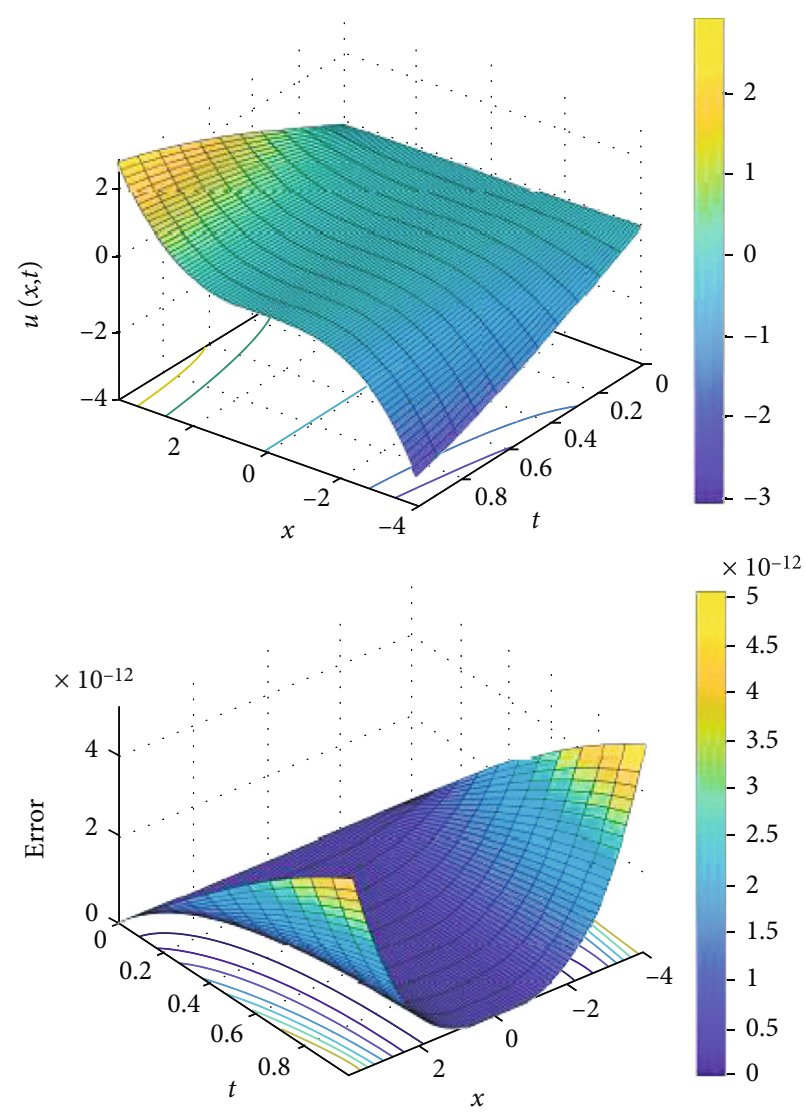

FIgure 2: Portrait of the numerical solution and the absolute error for Example 7 with $m=4, n=10$.

$$
\begin{aligned}
\mathfrak{D}_{\rho, \omega, 0^{+}}^{\gamma, \mu(t), v} \psi_{j}(t) & \simeq \sum_{r=1}^{n+1}\left[\frac{1}{\gamma_{j}} \sum_{k=0}^{n} b_{j, k} t_{r}^{n-\mu(t)-k} E_{\rho, n-\mu\left(t_{r}\right)-k+1}^{-\gamma}\left(\omega t_{r}^{\rho}\right)\right] \psi_{r}(t) \\
& =\sum_{r=1}^{n+1} \mathbb{P}_{j, r}^{\mu(t)} \psi_{r}(t), \quad j=1,2, \cdots, n+1 .
\end{aligned}
$$

So according to relations (22) and (23), we can get the following results:

$$
\begin{aligned}
{ }^{C} \mathfrak{D}_{\rho, \omega, 0^{+}}^{\gamma, \mu(t), v} \Lambda_{n}(t) & =\left[{ }^{C} \mathfrak{D}_{\rho, \omega, 0^{+}}^{\gamma, \mu(t), v} \psi_{1}(t),{ }^{C} \mathfrak{D}_{\rho, \omega, 0^{+}}^{\gamma, \mu(t), v} \psi_{2}(t), \cdots,{ }^{C} \mathfrak{D}_{\rho, \omega, 0^{+}}^{\gamma, \mu(t), v} \psi_{n+1}(t)\right]^{T} \\
& =\mathbb{E}^{\mu(t)} \Lambda_{n}(t), \mathbb{E}^{\mu(t)} \\
& =\left[\mathbb{q}_{j, r}^{\mu(t)}\right]_{(n+1) \times(n+1)}, \quad j=1,2, \cdots, n+1, r=1,2, \cdots, n+1, \\
\mathfrak{D}_{\rho, \omega, 0^{+}}^{\gamma, \mu(t), v} \Lambda_{n}(t) & =\left[\mathfrak{D}_{\rho, \omega, 0^{+}}^{\gamma, \mu(t), v} \psi_{1}(t), \mathfrak{D}_{\rho, \omega, 0^{+}}^{\gamma, \mu(t), v} \psi_{2}(t), \cdots, \mathfrak{D}_{\rho, \omega, 0^{+}}^{\gamma, \mu(t), v} \psi_{n+1}(t)\right]^{T} \\
& =\mathscr{E}^{\mu(t)} \Lambda_{n}(t), \mathscr{E}^{\mu(t)} \\
& =\left[\mathbb{P}_{j, r}^{\mu(t)}\right]_{(n+1) \times(n+1)}, \quad j=1,2, \cdots, n+1, r=1,2, \cdots, n+1 .
\end{aligned}
$$

Theorem 5. Suppose $u(x, t)$ is an exact solution and $u_{m}(x, t)$ is a numerical solution of Equation (1) in which $u(x, t), u_{m}$ 
TABle 3: Absolute error for different values of $m, n$ for Example 7.

\begin{tabular}{lcccc}
\hline$(x, t)$ & \multicolumn{2}{c}{$|u(x, t)-u *(x, t)|$} & \multicolumn{2}{c}{$|u(x, t)-u *(x, t)|$} \\
& $m=4, n=10$ & $m=10, n=20$ & $m=15, n=30$ & $2.3945 e-9$ \\
$(0.68,0.09)$ & $2.7916 e-8$ & $2.7082 e-8$ & $1.09125 e-9$ & $5.0958 e-10$ \\
$(0.86,0.18)$ & $1.0649 e-8$ & $5.4784 e-9$ & $1.9298 e-10$ & $9.0120 e-11$ \\
$(1.04,0.27)$ & $9.5832 e-9$ & $9.6885 e-10$ & $3.8569 e-11$ & $1.8011 e-11$ \\
$(1.31,0.09)$ & $1.9152 e-9$ & $1.9363 e-10$ & $1.8518 e-11$ & $8.6477 e-12$ \\
$(2.21,0.36)$ & $9.1957 e-10$ & $9.2968 e-11$ & $5.1607 e-12$ & $2.4099 e-12$ \\
$(3.11,0.45)$ & $2.5626 e-10$ & $1.9729 e-11$ & & \\
\hline
\end{tabular}
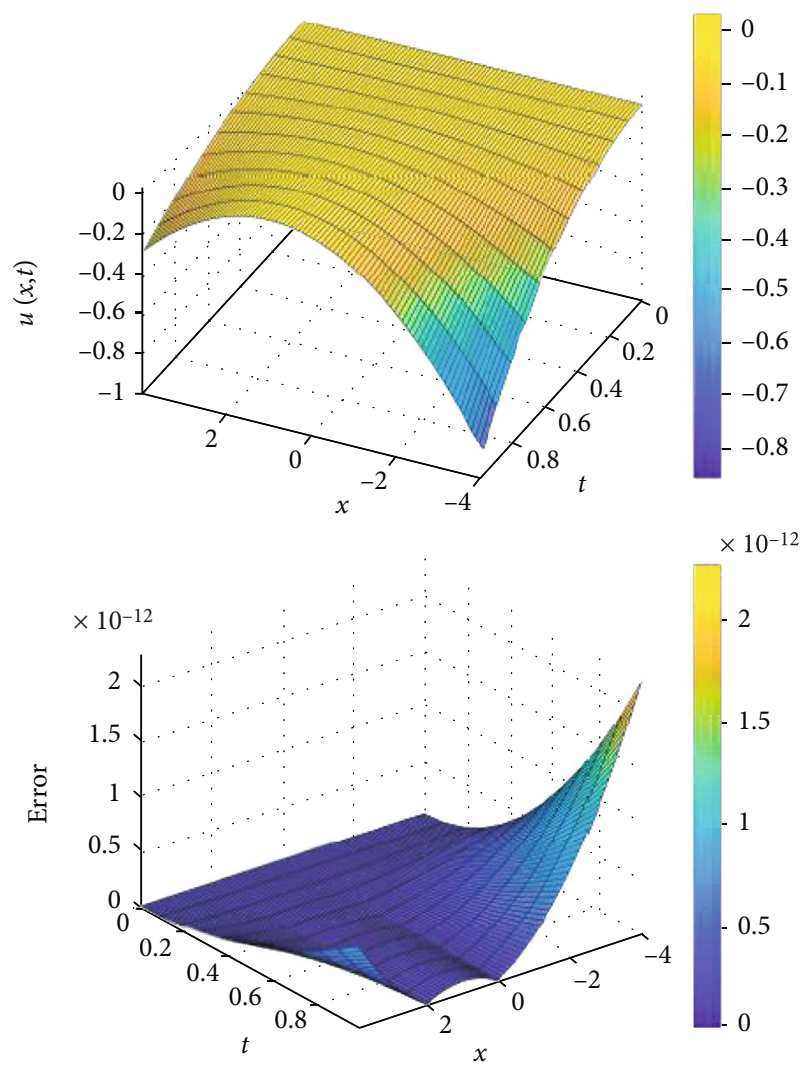

FIgURE 3: Portrait of the numerical solution and the absolute error for Example 8 with $m=4, n=10$.

$(x, t) \in C\left(\left[0, x_{\max }\right] \times\left[0, t_{\max }\right]\right)$ and $M=\max _{(x, t) \in\left[0, x_{\max }\right] \times\left[0, t_{\max }\right]}$ $\left|\left(\partial^{m+1} u(\rho, \varsigma)\right) / \partial t^{r} \partial x^{m+1-r}\right|$ exist. Then, the following relation holds:

$$
\left\|u(x, t)-u_{m}(x, t)\right\|_{2} \leq \frac{M\left(x_{\max }+t_{\max }\right)^{m+1} \sqrt{t_{\max } x_{\max }}}{(m+1) !}
$$

Proof. To prove, Taylor's expansion function $u(x, t)$ around a point $(0,0)$ can be written as

$$
\begin{aligned}
u(x, t)= & u(0,0)+\left(x \frac{\partial u(0,0)}{\partial x}+t \frac{\partial u(0,0)}{\partial t}\right)+\frac{1}{2 !}\left(x^{2} \frac{\partial^{2} u(0,0)}{\partial x^{2}}+t^{2} \frac{\partial^{2} u(0,0)}{\partial t^{2}}\right) \\
& +x t \frac{\partial^{2} u(0,0)}{\partial t \partial x}+\cdots+\frac{1}{m !}\left(x \frac{\partial u(0,0)}{\partial x}+t \frac{\partial u(0,0)}{\partial t}\right)^{m} \\
& +\frac{1}{(m+1) !}\left(x \frac{\partial u(\rho, \varsigma)}{\partial x}+t \frac{\partial u(\rho, \varsigma)}{\partial t}\right)^{m+1}
\end{aligned}
$$

We consider the following finite expansion as an approximation of the function $u(x, t)$ :

$$
\begin{aligned}
u(x, t) \simeq & u_{m}(x, t)=u(0,0)+\left(x \frac{\partial u(0,0)}{\partial x}+t \frac{\partial u(0,0)}{\partial t}\right) \\
& +\frac{1}{2 !}\left(x^{2} \frac{\partial^{2} u(0,0)}{\partial x^{2}}+t^{2} \frac{\partial^{2} u(0,0)}{\partial t^{2}}\right) \\
& +x t \frac{\partial^{2} u(0,0)}{\partial t \partial x}+\cdots+\frac{1}{m !}\left(x \frac{\partial u(0,0)}{\partial x}+t \frac{\partial u(0,0)}{\partial t}\right)^{m}
\end{aligned}
$$

Then, we have

$$
\begin{aligned}
\left\|u(x, t)-u_{m}(x, t)\right\|_{2}= & \left\|\frac{1}{(m+1) !}\left(x \frac{\partial u(\rho, \varsigma)}{\partial x}+t \frac{\partial u(\rho, \varsigma)}{\partial t}\right)^{m+1}\right\|_{2} \\
& \cdot\left[\int_{0}^{t_{\max }} \int_{0}^{x_{\max }}\left(\frac{1}{(m+1) !}\left(x \frac{\partial u(\rho, \varsigma)}{\partial x}+t \frac{\partial u(\rho, \varsigma)}{\partial t}\right)^{m+1}\right)^{2} d x d t\right]^{1 / 2},
\end{aligned}
$$

considering

$$
\left(x \frac{\partial u(\rho, \varsigma)}{\partial x}+t \frac{\partial u(\rho, \varsigma)}{\partial t}\right)^{m+1}=\sum_{r=0}^{m+1}\left(\begin{array}{c}
m \\
r
\end{array}\right) \frac{\partial^{m+1} u(\rho, \varsigma)}{\partial t^{r} \partial x^{m+1-r}} t^{r} x^{m+1-r}
$$

and we obtain 
TABLe 4: Absolute error for different values of $m, n$ for Example 8.

\begin{tabular}{lcccc}
\hline$(x, t)$ & \multicolumn{2}{c}{$|u(x, t)-u *(x, t)|$} & \multicolumn{2}{c}{$|u(x, t)-u *(x, t)|$} \\
& $m=4, n=10$ & $m=10, n=20$ & $2.8128 e-8$ & $8.5088 e-9$ \\
$(0.68,0.09)$ & $6.9725 e-7$ & $7.0321 e-8$ & $3.0723 e-9$ & $9.2937 e-10$ \\
$(0.86,0.18)$ & $7.6156 e-8$ & $7.6807 e-9$ & $3.1287 e-10$ & $9.4643 e-11$ \\
$(1.04,0.27)$ & $7.7555 e-9$ & $7.8218 e-10$ & $2.8325 e-10$ & $8.5685 e-11$ \\
$(1.31,0.09)$ & $7.0214 e-9$ & $7.0814 e-10$ & $1.4543 e-10$ & $4.3994 e-11$ \\
$(2.21,0.36)$ & $3.6050 e-9$ & $3.6359 e-10$ & $1.0817 e-10$ & $3.2724 e-11$ \\
$(3.11,0.45)$ & $2.6815 e-9$ & $2.7044 e-10$ & & \\
\hline
\end{tabular}
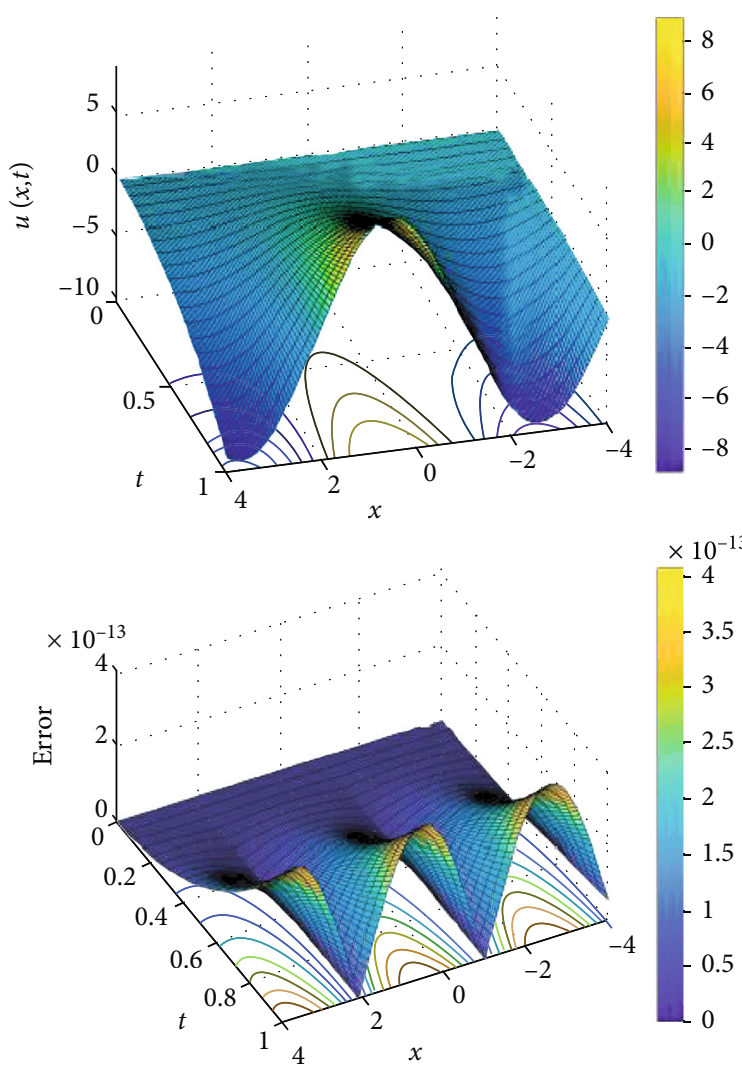

FIGURE 4: Portrait of the numerical solution and the absolute error for Example 9 with $m=4, n=10$.

$$
\begin{aligned}
\left\|u(x, t)-u_{m}(x, t)\right\|_{2} & \left.=\left[\int_{0}^{t_{\max }} \int_{0}^{x_{\max }}\left(\frac{1}{(m+1) !} \sum_{r=0}^{m+1}{ }^{m} r\right) \frac{\partial^{m+1} u(\rho, \varsigma)}{\partial t^{r} \partial x^{m+1-r}} t^{r} x^{m+1-r}\right)^{2} d x d t\right]^{1 / 2} \\
\leq & \max _{(x, t) \in\left[0, x_{\max }\right] \times\left[0, t_{\max }\right]} \mid \frac{\partial^{m+1} u(\rho, \varsigma)}{\partial t^{r} \partial x^{m+1-r} \mid} \\
& \cdot\left[\int_{0}^{t_{\max }} \int_{0}^{x_{\max }}\left(\frac{1}{(m+1) !} \sum_{r=0}^{m+1}\left(\begin{array}{c}
m \\
r
\end{array} t^{r} x^{m+1-r}\right)^{2} d x d t\right]^{1 / 2}\right. \\
= & \frac{M}{(m+1) !}\left(\int_{0}^{t_{\max }} \int_{0}^{x_{\max }}(x+t)^{2 m+2} d x d t\right)^{\frac{1}{2}} \\
\leq & \frac{M\left(x_{\max }+t_{\max }\right)^{m+1} \sqrt{t_{\max } x_{\max }}}{(m+1) !}
\end{aligned}
$$

For convergence of the function, sequence $u_{m}(x, t) \rightarrow u$ $(x, t)$ sufficient to show that the sequence $u_{m}(x, t)$ is a Cauchy sequence in space $C\left(\left[0, x_{\max }\right] \times\left[0, t_{\max }\right]\right)$. So, we have

$$
\begin{aligned}
\left\|u_{n}(x, t)-u_{m}(x, t)\right\|_{2} \leq & \left\|u(x, t)-u_{m}(x, t)\right\|_{2}+\left\|u(x, t)-u_{n}(x, t)\right\|_{2} \\
\leq & \frac{M_{1}\left(x_{\max }+t_{\max }\right)^{m+1} \sqrt{t_{\max } x_{\max }}}{(m+1) !} \\
& +\frac{M_{2}\left(x_{\max }+t_{\max }\right)^{n+1} \sqrt{t_{\max } x_{\max }}}{(n+1) !}, \quad M_{1}, M_{2}>0 .
\end{aligned}
$$

According to the convergence growth rate $\left(x_{\max }+t_{\max }\right)^{m+1} \ll(m+1)$ !, as $m, n \rightarrow+\infty$, we have $\| u_{n}(x$, $t)-u_{m}(x, t) \| \rightarrow 0$; therefore, the sequence $u_{m}(x, t)$ is a Cauchy sequence in space $C\left(\left[0, x_{\max }\right] \times\left[0, t_{\max }\right]\right)$. Since $C([0$, $\left.\left.x_{\max }\right] \times\left[0, t_{\max }\right]\right)$ is complete, then $u_{m}(x, t)$ converges. To prove the uniqueness of the solutions, we assume that $u, \bar{u}$ are two different solutions of $\lim _{m \rightarrow \infty} u_{m}(x, t)$; then, we have

$$
\|u(x, t)-\bar{u}(x, t)\|_{2} \leq\left\|u(x, t)-u_{m}(x, t)\right\|_{2}+\left\|\bar{u}-u_{m}(x, t)\right\|_{2} .
$$

Due to $\left\|u(x, t)-u_{m}(x, t)\right\| \rightarrow 0$ and $\left\|\bar{u}(x, t)-u_{m}(x, t)\right\|$ $\rightarrow 0$, we have $\|u(x, t)-\bar{u}(x, t)\|_{2} \rightarrow 0$, then $u=\bar{u}$.

\section{A Description of the Proposed Method for Solving the System}

In this section, using relations described in Section 3, we solve the proposed system stated in Section 1 which is introduced with (1). Using relation (15) and applying relations (24) and (25) on Equation (1), in this case, we obtain

$$
\begin{gathered}
{ }^{C} \mathfrak{D}_{\rho, \omega, 0^{+}}^{\gamma, \mu(t), v} u(x, t)+\mathfrak{D}_{\rho, \omega, 0^{+}}^{\gamma, \mu(t), v} u(x, t)=f(x, t, u(x, t)), \\
Y_{m}^{T}(x) \Delta \mathbb{E}^{\mu(t)} \Lambda_{n}(t)+Y_{m}^{T}(x) \Delta \mathscr{E}^{\mu(t)} \Lambda_{n}(t)=f(x, t, u(x, t)) .
\end{gathered}
$$


TABle 5: Absolute error for different values of $m, n$ for Example 9.

\begin{tabular}{lcccc}
\hline \multirow{2}{*}{$(x, t)$} & \multicolumn{2}{c}{$|u(x, t)-u *(x, t)|$} & \multicolumn{2}{c}{$|u(x, t)-u *(x, t)|$} \\
& $m=4, n=10$ & $m=10, n=20$ & $m=15, n=30$ & $2.3914 e-8$ \\
$(0.68,0.09)$ & $1.5851 e-7$ & $9.5658 e-8$ & $6.6331 e-8$ & $1.8148 e-8$ \\
$(0.86,0.18)$ & $1.2029 e-7$ & $7.2593 e-8$ & $6.5515 e-8$ & $1.2200 e-8$ \\
$(1.04,0.27)$ & $8.0869 e-8$ & $4.8802 e-8$ & $4.4043 e-8$ & $3.0692 e-9$ \\
$(1.31,0.09)$ & $2.0344 e-8$ & $1.2277 e-8$ & $1.1080 e-8$ & $2.6711 e-9$ \\
$(2.21,0.36)$ & $1.7705 e-8$ & $1.0684 e-8$ & $9.6429 e-9$ & $4.9952 e-10$ \\
$(3.11,0.45)$ & $3.3110 e-9$ & $1.9981 e-9$ & $1.8032 e-9$ & \\
\hline
\end{tabular}

Table 6: $C$-order of the presented method for Example 9.

\begin{tabular}{cccc}
\hline \multicolumn{2}{c}{$C$-order } & \multicolumn{2}{c}{$C$-order } \\
$m=4, n=10$ & $m=10, n=20$ & $m=15, n=30$ & $m=20, n=40$ \\
\hline 18.13 & 19.11 & 20.34 & 21.54 \\
\hline
\end{tabular}

Using relation (15) for the function $f(x, t)$, in this case, the following relation is obtained:

$$
\begin{gathered}
Y_{m}^{T}(x) \Delta \mathbb{E}^{\mu(t)} \Lambda_{n}(t)+Y_{m}^{T}(x) \Delta \mathscr{E}^{\mu(t)} \Lambda_{n}(t)=Y_{m}^{T}(x) M \Lambda_{n}(t), \\
Y_{m}^{T}(x) \Delta\left(\mathbb{E}^{\mu(t)}+\mathscr{E}^{\mu(t)}\right) \Lambda_{n}(t)=Y_{m}^{T}(x) M \Lambda_{n}(t), \\
Y_{m}^{T}(x)\left(\Delta\left(\mathbb{E}^{\mu(t)}+\mathscr{E}^{\mu(t)}\right)-M\right) \Lambda_{n}(t) \triangleq Y_{m}^{T}(x) W \Lambda_{n}(t) \simeq 0,
\end{gathered}
$$

where $M=\left[f\left(x_{i}, t_{j}, u\left(x_{i}, t_{j}\right)\right)\right]_{(m+1) \times(n+1)}$ and $W=\left(\Delta\left(\mathbb{E}^{\mu(t)}+\right.\right.$ $\left.\mathscr{E}^{\mu(t)}\right)-M$ ) and the initial condition for this equation is defined by

$$
\begin{gathered}
u(x, 0)=d \simeq Y_{m}^{T}(x) \times \Delta \times \Lambda_{n}(0)=Y_{m}^{T}(x) \mathbb{d}, \\
Y_{m}^{T}(x)\left(\Delta \Lambda_{n}(0)-\mathbb{d}\right) \triangleq Y_{m}^{T}(x) \Xi \simeq 0,
\end{gathered}
$$

Here, $\quad d(x)=\sum_{i=1}^{m+1} d\left(x_{i}\right) \varphi_{i}(x) \triangleq \mathbb{d}^{T} Y_{m}(x), \mathbb{d}=$ $\left[d\left(x_{1}\right), d\left(x_{2}\right), \cdots, d\left(x_{m+1}\right)\right]^{T}$, and $\Xi=\left(\Delta \Lambda_{n}(0)-\mathbb{d}\right)$ are considered. By solving the following equations, the unknown function $u(x, t)$ is determined:

$$
\begin{gathered}
W_{i, j}=0, \quad i=2,3, \cdots, m, j=2,3, \cdots, n, \\
(\Xi)_{i}=0, \quad i=1,2,3, \cdots, m+1 .
\end{gathered}
$$

\section{Show Numerical Examples Using the Proposed Method}

In this section, we show the numerical results of the suggested method on four examples to illustrate the efficiency and accuracy of our scheme, where each of these examples is taken from various articles and all these examples using the method described in this article have been resolved for different values of a continuous function $\mu(t)$ and $m, n$. Here, obtained numerical results of some test problems are showed to illustrate the computational efficiency and applicability of the reported method. All numerical examples are done by applying MATLAB (R2020b) software. Also, the convergence order ( $C$-order) of the depicted method is calculated by the following relation:

$$
C \text {-order }=\frac{\left|\log \left(E_{1} / E_{2}\right)\right|}{\log \left(\left(\left(m_{2}+1\right)\left(m_{2}+1\right)\right) /\left(\left(m_{1}+1\right)\left(m_{1}+1\right)\right)\right)} .
$$

Here, $E_{1}$ and $E_{2}$ show the first and the second values of the maximum absolute error (MAE) provided by the proposed method, respectively. Moreover, $\left(m_{i}+1\right)\left(m_{i}+1\right)$ for $i=1,2$ are the number of the basis functions used in the first and the second implementations, respectively. Here, the absolute error which is the difference between a numerical and exact solution is defined by

$$
E=\left|u_{\text {exact }}-u_{\text {numerical }}\right|
$$

Example 6. Let $\mu(t)=(6 / 10)-(3 / 10) \sin (\pi t)$. Then, we consider the following linear fractional system of orders $\mu(t)$ :

$$
\begin{aligned}
& { }^{C} \mathfrak{D}_{\rho, \omega, 0^{+}}^{\gamma, \mu(t), v} u(x, t)+\frac{1}{2} \mathfrak{D}_{\rho, \omega, 0^{+}}^{\gamma, \mu(t), v} u(x, t)=f(x, t, u(x, t)), \\
& u(x, 0)=e^{-x},
\end{aligned}
$$

where $f(x, t, u(x, t))$ is given by

$$
\begin{aligned}
f(x, t)= & e^{-(x+t)}\left(e^{-(x+t)}-\sin (t)-1\right) \\
& +\left[\sum_{k=0}^{\infty}(-1)^{k} t^{k+1-\mu(t)} E_{\rho, k+2-\mu(t)}^{-\gamma}\left(\omega t^{\rho}\right)\right] e^{-x} \\
& +\frac{1}{2}\left[\sum_{k=0}^{\infty}(-1)^{k} t^{k+1-\mu(t)} E_{\rho, k+1-\mu(t)}^{-\gamma}\left(\omega t^{\rho}\right)\right] e^{-x} .
\end{aligned}
$$

The exact solution is $u(x, t)=e^{-(x+t)}$. The upper part in Figure 1 illustrates the numerical solution of Example 6 with $m=4$ and $n=10$, and the lower part shows the absolute error of this example. Table 1 shows the absolute error values for different values of $m, n$. Also, The values of the $C$-order of the presented method are listed in Table 2. From the obtained 
numerical results in Table 1, we see that the proposed method is an effective and good tool for solving this problem.

Example 7. Let $\mu(t)=(\sin (10 \pi t)) / 6+(2 / 3)$. Then, we state the following nonlinear fractional system:

$$
\begin{gathered}
C_{\mathfrak{D}_{\rho, \omega, 0^{+}}^{\gamma, \mu(t), v}} u(x, t)+\frac{1}{2} \mathfrak{D}_{\rho, \omega, 0^{+}}^{\gamma, \mu(t), v} u(x, t)=f(x, t, u(x, t)), \\
u(x, 0)=0,
\end{gathered}
$$

where $f(x, t, u(x, t))$ is given by

$$
\begin{aligned}
f(x, t, u(x, t))= & \sin (u(x, t))-\left[\sum_{k=0}^{\infty}(-1)^{k} t^{2 k+2-\mu(t)} E_{\rho, 2 k+3-\mu(t)}^{-\gamma}\left(\omega t^{\rho}\right)\right] x^{3} \\
& -\frac{1}{2}\left[\sum_{k=0}^{\infty}(-1)^{k} t^{2 k+2-\mu(t)} E_{\rho, 2 k+3-\mu(t)}^{-\gamma}\left(\omega t^{\rho}\right)\right] x^{3} \\
& -\left(\sin \left(x^{3} \sin (t)\right)+6 x t^{3} \sin (t)+x^{3} \cos (t)\left(1+e^{-x t}\right)\right) .
\end{aligned}
$$

The analytical solution for this question is $u(x, t)=x^{3}$ $\sin (t)$. The upper part in Figure 2 illustrates the numerical solution of Example 7 with $m=4$ and $n=10$, and the lower part shows the absolute error of this example. Table 3 shows the absolute error values for different values of $m, n$.

Example 8. We consider a fractional system of orders $\mu(t)$ $=t^{2}-t+0.8$ as follows:

$$
\begin{gathered}
C_{\mathfrak{D}_{\rho, \omega, 0^{+}}^{\gamma, \mu(t), v}} u(x, t)-\mathfrak{D}_{\rho, \omega, 0^{+}}^{\gamma, \mu(t), v} u(x, t)+u(x, t)=f(x, t), \\
u(x, 0)=0,
\end{gathered}
$$

where $f(x, t)$ is given as

$$
f(x, t)=\frac{2 x(2-x)\left(t^{2}-t\right)}{\ln (t)}+t^{2}\left(2+2 x-x^{2}\right)
$$

where for this problem, analytical solution is $u(x, t)=t^{2}(2 x$ $\left.-x^{2}\right)$.The upper part in Figure 3 illustrates the numerical solution of Example 8 with $m=4, n=10$, and the lower part shows the absolute error of this example. Table 4 shows the absolute error values for different values of $m, n$.

Example 9. We consider a fractional system as follows:

$$
\begin{gathered}
{ }^{C} \mathfrak{D}_{\rho, \omega, 0^{+}}^{\gamma, \mu(t), v} u(x, t)+\mathfrak{D}_{\rho, \omega, 0^{+}}^{\gamma, \mu(t), v} u(x, t)-\left(\frac{\partial^{2}}{\partial x^{2}}+\frac{\partial^{2}}{\partial t^{2}}\right) u(x, t)=f(x, t), \\
u(x, 0)=0, \mu(t)=\frac{5}{10}-\frac{3 e^{-t}}{10}
\end{gathered}
$$

where $f(x, t)$ is given as

$$
f(x, t)=2^{l} t^{l-1}\left(\frac{(t-1) \Gamma(1+l)}{\ln (t)}+t\right) \sin (x+1), l>0,
$$

where for this problem, analytical solution is $u(x, t)=2^{l} t^{l}$ $\sin (x+1)$. The upper part in Figure 4 illustrates the numerical solution of Example 9 with $m=4$ and $n=10$, and the lower part shows the absolute error of this example. Table 5 shows the absolute error values for different values of $m, n$. Also, The values of the $C$-order of the presented method are listed in Table 6.

\section{Data Availability}

No data were used to support the study.

\section{Conflicts of Interest}

The author declares that there are no competing interests.

\section{Authors' Contributions}

All authors read and approved the final draft.

\section{References}

[1] A. Carpinteri and F. Mainardi, Fractals and Fractional Calculus in Continuum Mechanics, Springer, 2014.

[2] S. K. Panda, T. Abdeljawad, and C. Ravichandran, "A complex valued approach to the solutions of Riemann-Liouville integral, Atangana-Baleanu integral operator and non-linear telegraph equation via fixed point method," Chaos, Solitons \& Fractals, vol. 130, article 109439, 2020.

[3] I. Podlubny, Fractional Differential Equations: An Introduction to Fractional Derivatives, Fractional Differential Equations, to Methods of Their Solution and Some of Their Applications, Elsevier Academic Press, San Diego, 1999.

[4] J. A. Sabatier, O. P. Agrawal, and J. T. Machado, Advances in Fractional Calculus, Springer, Dordrecht, 2007.

[5] C. Ball, C. Cabrera, M. Adams, and P. A. Gourdain, "Radial basis functions as generators of the inviscid Burger's equation," Bulletin of the American Physical Society, vol. 22, p. 64, 2019.

[6] P.-L. Ma, S.-F. Tian, L. Zou, and T.-T. Zhang, "The solitary waves, quasi-periodic waves and integrability of a generalized fifth-order Korteweg-de Vries equation," Waves in Random and Complex Media, vol. 29, no. 2, pp. 247-263, 2018.

[7] M. U. Farooq, M. S. Khan, and A. Hajizadeh, "Flow of viscous fluid over an infinite plate with Caputo-Fabrizio derivatives," Open Journal of Mathematical Sciences, vol. 3, no. 1, pp. 115120, 2019.

[8] E. K. Akgül, "Solutions of the linear and nonlinear differential equations within the generalized fractional derivatives," Chaos: An Interdisciplinary Journal of Nonlinear Science, vol. 29, no. 2, article 023108, 2019.

[9] E. A. Az-Zóbi, "Exact analytic solutions for nonlinear diffusion equations via generalized residual power series method," International Journal of Mathematics and Computer Science, vol. 14, no. 1, pp. 69-78, 2019.

[10] D. Baleanu, "Approximate solutions for solving nonlinear variable-order fractional Riccati differential equations," 
Nonlinear Analysis: Modelling and Control, vol. 24, no. 2, pp. 176-188, 2019.

[11] K. Sun and M. Zhu, "Numerical algorithm to solve a class of variable order fractional integral- differential equation based on Chebyshev polynomials," Mathematical Problems in Engineering, vol. 2015, Article ID 902161, 10 pages, 2015.

[12] B. P. Moghaddam and J. A. T. Machado, "A computational approach for the solution of a class of variable-order fractional integro-differential equations with weakly singular kernels," Fractional Calculus and Applied Analysis, vol. 20, no. 4, pp. 1023-1042, 2017.

[13] A. H. Bhrawy and M. A. Zaky, "Numerical simulation for two-dimensional variable-order fractional nonlinear cable equation," Nonlinear Dynamics, vol. 80, no. 1-2, pp. 101116, 2015.

[14] Y. M. Chen, Y. Q. Wei, D. Y. Liu, and H. Yu, "Numerical solution for a class of nonlinear variable order fractional differential equations with Legendre wavelets," Applied Mathematics Letters, vol. 46, pp. 83-88, 2015.

[15] A. H. Bhrawy and M. Zaky, "An improved collocation method for multi-dimensional space-time variable-order fractional Schrodinger equations," Applied Numerical Mathematics, vol. 111, pp. 197-218, 2017.

[16] A. H. Bhrawy and M. A. Zaky, "Highly accurate numerical schemes for multi-dimensional space variable-order fractional Schrodinger equations," Computers \& Mathematics with Applications, vol. 73, no. 6, pp. 1100-1117, 2017.

[17] A. Mahmoud, I. G. Ameen, and A. A. Mohamed, "A new operational matrix based on jacobi wavelets for a class of variableorder fractional differential equations," Proceedings of the Romanian Academy Series A, vol. 18, no. 4, pp. 315-322.

[18] M. A. Zaky, S. S. Ezz-Eldien, E. H. Doha, J. A. Tenreiro Machado, and A. H. Bhrawy, "An efficient operational matrix technique for multidimensional variable-order time fractional diffusion equations," Journal of Computational and Nonlinear Dynamics, vol. 11, no. 6, 2016.

[19] M. A. Zaky, A. S. Hendy, A. A. Alikhanov, and V. G. Pimenov, "Numerical analysis of multi-term time-fractional nonlinear subdiffusion equations with time delay: what could possibly go wrong," Communications in Nonlinear Science and Numerical Simulation, vol. 96, 2020.

[20] A. H. Bhrawy, M. A. Zaky, and J. F. Alzaidy, "Two shifted Jacobi-Gauss collocation schemes for solving twodimensional variable-order fractional Rayleigh-Stokes problem," Adv. Difference Equ., vol. 2016, no. 1, 2016.

[21] A. Atangana and R. T. Alqahtani, "New numerical method and application to Keller-Segel model with fractional order derivative," Chaos, Solitons \& Fractals, vol. 116, pp. 14-21, 2018.

[22] A. A. Alikhanov, "Numerical methods of solutions of boundary value problems for the multi-term variable-distributed order diffusion equation," Applied Mathematics and Computation, vol. 268, pp. 12-22, 2015.

[23] E. H. Doha, M. A. Abdelkawy, A. Z. Amin, and D. Baleanu, "Spectral technique for solving variable-order fractional Volterra integro-differential equations," Numerical Methods for Partial Differential Equations, vol. 34, no. 5, pp. 1659-1677, 2018.

[24] S. Sadeghi, H. Jafari, and S. Nemati, "Operational matrix for Atangana-Baleanu derivative based on Genocchi polynomials for solving FDEs," Chaos, Solitons \& Fractals, vol. 135, article 109736, 2020.
[25] Y. Xu and E. V. Suat, "A finite difference technique for solving variable-order fractional integro-differential equations," Bulletin of the Iranian Mathematical Society, vol. 40, no. 3, pp. 699$712,2014$.

[26] M. Yi, J. Huang, and L. Wang, "Operational matrix method for solving variable order fractional integro-differential equations," CMES-Computer Modeling in Engineering and Sciences, vol. 96, pp. 361-377, 2013.

[27] M. Zayernouri and G. E. Karniadakis, "Exponentially accurate spectral and spectral element methods for fractional ODEs," Journal of Computational Physics, vol. 257, pp. 460-480, 2014.

[28] R. Garra, R. Gorenflo, F. Polito, and Ž. Tomovski, "HilferPrabhakar derivatives and some applications," Applied mathematics and computation, vol. 242, pp. 576-589, 2014.

[29] R. Gorenflo, A. A. Kilbas, F. Mainardi, and S. V. Rogosin, Mittag-Leffler Functions, Related Topics and Applications, Springer, Berlin, 2014.

[30] J. Hristov, "Response functions in linear viscoelastic constitutive equations and related fractional operators," Mathematical Modelling of Natural Phenomena, vol. 14, no. 3, p. 305, 2019.

[31] R. Garra and R. Garrappa, "The Prabhakar or three parameter Mittag-Leffler function: theory and application," Communications in Nonlinear Science and Numerical Simulation, vol. 56, pp. 314-329, 2018.

[32] R. Garrappa, F. Mainardi, and M. Guido, "Models of dielectric relaxation based on completely monotone functions," Fractional Calculus and Applied Analysis, vol. 19, no. 5, pp. 1105-1160, 2016.

[33] A. Giusti and I. Colombaro, "Prabhakar-like fractional viscoelasticity," Communications in Nonlinear Science and Numerical Simulation, vol. 56, pp. 138-143, 2018.

[34] M. D'Ovidio and F. Polito, "Fractional diffusion-telegraph equations and their associated stochastic solutions," Theory of Probability \& Its Applications, vol. 62, no. 4, pp. 552-574, 2018.

[35] S. C. Pandey, "The Lorenzo-Hartley's function for fractional calculus and its applications pertaining to fractional order modelling of anomalous relaxation in dielectrics," Computational and Applied Mathematics, vol. 37, no. 3, pp. 26482666, 2018.

[36] A. A. Kilbas, M. Saigo, and R. K. Saxena, "Generalized MittagLeffler function and generalized fractional calculus operators," Integral Transforms and Special Functions, vol. 15, no. 1, pp. 31-49, 2004.

[37] F. Polito and Ž. Tomovski, "Some properties of Prabhakartype fractional calculus operators," 2015, http://arxiv.org/abs/ 1508.03224.

[38] M. H. Heydari, "A new direct method based on the Chebyshev cardinal functions for variable- order fractional optimal control problems," Journal of the Franklin Institute, vol. 355, no. 12, pp. 4970-4995, 2018.

[39] K.-H. Pho, M. H. Heydari, B. A. Tuan, and M. R. Mahmoudi, "Numerical study of nonlinear 2D optimal control problems with multi-term variable-order fractional derivatives in the Atangana-Baleanu-Caputo sense," Chaos, Solitons \& Fractals, vol. 134, 2020. 